\title{
IRREDUCIBLE MARKOV OPERATORS ON $C(S)$
}

\section{BENTON JAMISON ${ }^{1}$}

1. Let $S$ be a compact Hausdorff space, $C(S)$ the Banach space of all real-valued continuous functions on $S$ with the supremum norm. A linear operator $T$ on $C(S)$ is said to be a Markov operator if $T(1)=1$ and if $f \geqq 0$ implies $T f \geqq 0$. It is known [5] that such an operator is of the form

$$
(T f)(x)=\int f(y) P(x, d y), \quad x \in C(S),
$$

where, for each $x \in S, P(x, \cdot)$ is a regular probability measure on the $\sigma$-field $\Sigma$ of Borel subsets of $S$, and where the map $x \rightarrow P(x, \cdot)$ is continuous relative to the weak ${ }^{*}$ topology on $C^{*}(S)$. The operator $T$ is called irreducible if for each nonnegative but somewhere positive $f \in C(S)$ and for each $x \in S$ there is an $n$ such that $\left(T^{n} f\right)(x)>0$. This is the case if and only if for each open set $U$ and each $x$ there is an $n$ for which $P^{n}(x, U)>0$. If $T$ is irreducible, then $T f=f$ and $f \in C(S)$ if and only if $f$ is constant on $S$ (Theorem 2.4 of [4]). A nonempty $E \in \Sigma$ is said to be stochastically closed if $P(x, E)=1$ for each $x \in E$. If $T$ is irreducible then no proper closed subset of $S$ is stochastically closed. We say that $T$ is strongly (weakly) almost periodic if for each $f \in C(S)$ the set $\left\{T^{n} f: n=1,2, \cdots\right\}$ has a cluster point relative to the strong (weak) topology on $C(S)$ (see [1], [5]).

The purpose of this note is to prove the following

Theorem. Suppose $T$ is an irreducible Markov operator on $C(S)$, where $S$ is compact Hausdorff. Let $f \in C(S)$. If

$$
\lim _{n}\left(T^{n} f\right)(x)=0, \quad x \in S,
$$

then $\left\|T^{n} f\right\| \rightarrow 0$.

We give the proof in $\$ 3$. Rosenblatt, sharpening results of deLeeuw and Glicksberg [1], has shown [5, p. 217], that if $T$ is irreducible and weakly almost periodic then

$$
C(S)=C_{p} \oplus C_{0},
$$

Received by the editors August 2, 1968, and, in revised form, August 2, 1969.

1 This research was partially supported by the Air Force Office of Scientific Research. 
where $C_{p}$ is the closed linear span of the eigenfunctions corresponding to the unimodular eigenvalues of $T$ and where

$$
C_{0}=\left\{f:\left(T^{n} f\right)(x) \rightarrow 0, \forall x \in S\right\} .
$$

Since $\left\{T^{n} f\right\}$ is conditionally compact in the strong topology for each $f \in C_{0}$, our theorem has the following

Corollary. An irreducible weakly almost periodic Markov operator on $C(S)$ is strongly almost periodic.

2. In this section we assume that $S$ is compact metric with metric $d$. Let $\left\{f_{n}\right\} \subset C(S),\left\|f_{n}\right\| \leqq k<\infty$ for all $n$. For each $x \in S$ we define

$$
O(x)=\lim _{m} \limsup _{n}\left(\sup \left\{\left|f_{n}(y)\right|: d(x, y) \leqq 1 / m\right\}\right) \text {. }
$$

It is not hard to see that if $n_{k} \rightarrow \infty$ and $x_{k} \rightarrow x$ then lim sup ( $\sup \left|f_{n_{k}}\left(x_{k}\right)\right|$ ) $\leqq O(x)$, and also that there is an $n_{k} \rightarrow \infty$ and $x_{k} \rightarrow x$ with $\left|f_{n_{k}}\left(x_{k}\right)\right|$ $\rightarrow O(x)$. We list three of the properties of $O(x)$ as a function on $S$, leaving the rather routine verifications of the first two to the reader.

A. $O(x)$ is upper semicontinuous.

In particular, $O(x)$ assumes its least upper bound $\alpha$.

B. $\left\|\left(\left|f_{n}\right| \vee \alpha\right)-\alpha\right\| \rightarrow 0$ as $n \rightarrow \infty$.

C. If $f_{n}(x) \rightarrow 0$ for each $x \in S,\{x: O(x)>0\}$ is a set of the first category.

Proof of C. Assume that $f_{n} \rightarrow 0$ pointwise on $S$. For each $j=1$, $2, \cdots$, let

$$
O_{j}=\{x: O(x) \geqq 1 / j\} .
$$

Each $O_{j}$ is closed by virtue of (A). Fix $j$ and suppose that $O_{j}$ has nonempty interior $U$. Then there is a closed $V \subset U$ with nonempty interior. Fix each $j=1,2, \cdots$, let

$$
S_{i}=\left\{x: x \in V \text { and }\left|f_{k}(x)\right| \leqq 1 /(2 j) \text { for all } k \geqq i\right\} \text {. }
$$

Each $S_{i}$ is a closed subset of $V$. Since $f_{n} \rightarrow 0$ on $S$ the $S_{i}$ 's cover $V$. But $V$ itself is a complete metric space, so at least one of the $S_{\imath}$ 's must have a nonempty interior $W$. On the one hand $W \subset O_{j}$ $=\{x: O(x) \geqq 1 / j\}$, and on the other hand it is clear that $W$ $C\{x: O(x) \leqq 1 /(2 j)\}$. This is a contradiction. It follows that each of the $O^{\prime}{ }_{j}$ 's has empty interior. Since $\{x: O(x)>0\}$ is the union of the $O_{j}$ 's, it is a set of the first category.

3. This section is devoted to the proof of the theorem of $\$ 1$. Assume that $T^{n} f \rightarrow 0$ pointwise. We first consider the case where $S$ is 
compact metric. Let $\left\{T^{n} f\right\}$ be the sequence $\left\{f_{n}\right\}$ of the preceding section, with $\alpha=\sup _{x} O(x)$. Suppose $\alpha>0$. Let $E=\{x: O(x)=\alpha\}$. Since $E=\{x: O(x) \geqq \alpha\}, E$ is closed and $\neq \varnothing$ by virtue of (A). Claim: $E$ is stochastically closed.

This is trivial if $E=S$. Otherwise, let $x \in E$. There are sequences $x_{k} \rightarrow x$ and $n_{k} \rightarrow \infty$ with $\left|T^{n_{k}+1} f\left(x_{k}\right)\right| \rightarrow \alpha$. Now suppose $P\left(x, E^{c}\right)>0$. It is easy to show that there is a closed set $F \subset E^{c}$ and a number $p>0$ such that $P\left(x_{k}, F\right) \geqq p$ for all sufficiently large $k$. Let $\beta=\sup _{x \in F} O(x)$. Since $F$ is closed, (A) yields a $z \in F$ with $O(z)=\beta$; since $F \subset E^{c}, \beta<\alpha$. Let $2 \epsilon=\alpha-\beta$. Suppose $\left|\left(T^{n}{ }_{k f}\right)(y)\right| \vee(\alpha-\epsilon) \rightarrow \alpha-\epsilon$ as $k \rightarrow \infty$ uniformly for $y \in F$. We have

$$
\left(T^{n_{k}+1} f\right)\left(x_{k}\right)=\int_{\boldsymbol{F}}\left(T^{n_{k}} f\right)(y) P\left(x_{k}, d y\right)+\int_{F^{c}}\left(T^{n_{k}} f(y)\right) P\left(x_{k}, d y\right) .
$$

However, $\left|T^{n} f\right| \vee \alpha \rightarrow \alpha$ uniformly on $S$, hence on $F^{c}$ (by virtue of (B)). Since $P\left(x_{k}, F\right) \geqq p$ for all sufficiently large $k$, it would follow that

$$
\alpha=\lim \left|T^{n_{k}+1} f\left(x_{k}\right)\right| \leqq(\alpha-\epsilon) p+\alpha(1-p)=\alpha-p \epsilon<\alpha,
$$

which is, of course, a contradiction. Thus we can find a subsequence $\left\{k_{j}\right\}$ and a sequence $y_{j} \in F$ such that $\left|T^{n_{k}} j f\left(y_{j}\right)\right|>\alpha-\epsilon$. We may assume without loss of generality that $\left\{y_{j}\right\}$ converges, its limit $y$ being necessarily in $F$. But then $O(y) \geqq \alpha-\epsilon>\alpha-2 \epsilon=\beta$, which is an impossibility since $y \in F$ and $\sup _{x \in F} O(x)=\beta$. This shows that $P\left(x, E^{c}\right)=0$. Since $x$ is arbitrary, this establishes that $E$ is stochastically closed.

Since $E$ is both topologically and stochastically closed, $E=S$ by irreducibility. But this contradicts (C). Thus $\alpha=0$, whence $\left\|T^{n} f\right\| \rightarrow 0$ by virtue of (B). let

Now suppose $S$ is not metric. Let $A_{1}=\{f\}$. For each $n=1,2, \cdots$,

$$
A_{2 n}=A_{2 n-1} \cup\left\{T g: g \in A_{2 n-1}\right\},
$$

and let $A_{2 n+1}$ be the union of $A_{2 n}$ with the class of all finite products of members of $A_{2 n}$. Let $A_{\infty}=\{1\} \cup \cup_{n=1}^{\infty} A_{n}$. Then $A_{\infty} A_{\infty} \subset A_{\infty}$. Let $A_{0}$ be the collection of all finite rational linear combinations of members of $A_{\infty}$. Then $A_{0} A_{0} \subset A_{0}, T A_{0} \subset A_{0}$, and $A_{0}$ is closed under rational linear combinations, Let $A$ be the uniform closure of $A$. Then $A$ is a closed subalgebra of $C(S)$ containing $T^{n} f$ for each $n=1,2, \cdots$, and $T A \subset A$. The level sets of $A$ from a set $\widetilde{S}$ which is compact Hausdorff relative to its quotient topology. To each $f \in C(S)$ which is constant on the level sets of $A$ there corresponds an $\tilde{f} \in C(\tilde{S})$, and vice versa. 
To $A$ corresponds a closed subalgebra $\tilde{A}$ of $C(\tilde{S})$ which separates points and contains 1 , so $\tilde{A}=C(\widetilde{S})$ by the Stone-Weierstrass Theorem. Since $A$ is separable, so is $C(\tilde{S})$, hence $\tilde{S}$ is metrizable. Since $T A \subset A$, the restriction of $T$ to $A$ lifts to a Markov operator $\widetilde{T}$ on $C(\tilde{S})$ for which $\tilde{T}^{n} \tilde{f}=T^{n} f$. The irreducibility of $\widetilde{T}$ follows from that of $T$. Now, $T^{n} f \rightarrow 0$ pointwise $\Rightarrow \widetilde{T}^{n} \tilde{f} \rightarrow 0$ pointwise $\Rightarrow\left\|\widetilde{T}^{n} \tilde{f}\right\| \rightarrow 0$ by the proof for the metric case, so $\left\|T^{n} f\right\|=\left\|\tilde{T}^{n} \tilde{f}\right\| \rightarrow 0$. This completes the proof of the theorem.

4. Suppose $S$ is the one point compactification of the integers, the latter having the discrete topology. As usual, $\infty$ denotes the point at infinity. Suppose $P(j,\{j+1\})=1, j=0, \pm 1, \cdots$, and that $P(\infty,\{\infty\})$ $=1$. Then $\left(T^{n} f\right)(x)$ converges pointwise to $f(\infty)$ for each $f \in C(S)$, so $T$ is weakly almost periodic. If $f(0)=1$ and $f(x)=0$ for $x \in S-\{0\}$, then $f(\infty)=0$, but $\left(T^{n} f\right)(-n)=1$. Thus $T$ is not strongly almost periodic. This shows that the condition of irreducibility is essential for the truth of corollary.

Suppose that $S$ is locally compact but not compact, and that $C(S)$ is the space of all bounded continuous functions. Then even if $T$ is irreducible and $T^{n} f \rightarrow 0$ pointwise, $\left\{T^{n} f\right\}$ need not converge uniformly. To see this, let $S$ be the nonnegative integers with the discrete topology. Then if $T$ is a Markov operator on $C(S)$, there is a matrix $P$ on $S \times S$ such that $\left(T^{n} f\right)(i)=\Sigma j^{\infty}=0 p^{(n)}(i, j) f(j), i \in S$, where $p^{(n)}(i, j)$ is the $(i, j)$ th entry of $P^{n}$. Assume that $P$ is irreducible, periodic, and positive recurrent (see [3, Chapter 13], for terminology). Then there is a probability measure $\mu$ on $S$ such that $p^{(n)}(i, j) \rightarrow \mu(j)$ as $n \rightarrow \infty$. It follows easily that $T^{n} f \rightarrow \int f d \mu$ pointwise for each $f \in C(S)$. Were the convergence uniform for each such $f$, we would have in particular $p^{(n)}(i, 0) \rightarrow \mu(0)$ uniformly in $i$. This is impossible if, for example, $p^{(n)}(n, 0)=1$ but $p^{(m)}(n, 0)=0$ for $m \leqq n$. For an example of such a $P$ which is at the same time irreducible, aperiodic, and null recurrent, see Problem 6 on p. 376 of [3]. As a matter of fact, it is not difficult to see that if $p^{(n)}(i, 0) \rightarrow \mu(0)>0$ uniformly in $i$ then Doeblin's condition holds (see p. 192 of [2]). This is a very strong condition on $P$ and, if it holds, the convergence is uniformly exponential; that is, there is a $0<\rho<1$ and a $K>0$ such that $\left|p^{(n)}(i, j)-\mu(j)\right| \leqq A \rho^{n}$ for all $i, j$ and $n$ (see [2, pp. 207-208]).

\section{REFERENCES}

1. K. de Leeuw and I. Glicksberg, Applications of almost periodic compactifications, Acta Math. 105 (1961), 63-97. MR 24 \#A1632. 
2. J. R. Doob, Stochastic processes, Wiley, New York and Chapman \& Hall, London, 1953. MR 15, 445.

3. W. Feller, $A n$ introduction to probability theory and its applications. Vol. 1, 2nd ed., Wiley, New York and Chapman \& Hall, London, 1957. MR 19, 466.

4. B. Jamison, Asymptotic behavior of successive iterates of continuous functions under a Markov operator, J. Math. Anal. Appl. 9 (1964), 203-214. MR 29 \#6295.

5. M. Rosenblatt, Equicontinuous Markov operators, Teor. Verojatnost. i Primenen 9 (1964), 205-222. MR $30 \# 1549$.

University OF MinNEsota 